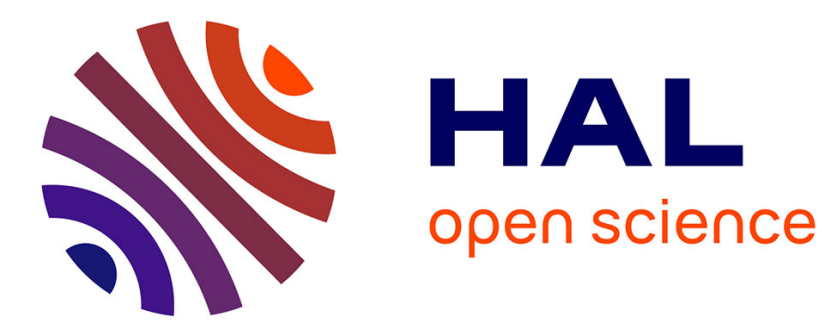

\title{
Microporous electrostrictive materials for vibrational energy harvesting
}

Mickaël Pruvost, Wilbert Smit, Cécile Monteux, Philippe Poulin, Annie Colin

\section{To cite this version:}

Mickaël Pruvost, Wilbert Smit, Cécile Monteux, Philippe Poulin, Annie Colin. Microporous electrostrictive materials for vibrational energy harvesting. Multifunctional Materials, 2018, 1 (1), 10.1088/2399-7532/aab2ff . hal-01903734

\section{HAL Id: hal-01903734 \\ https://hal.science/hal-01903734}

Submitted on 24 Oct 2018

HAL is a multi-disciplinary open access archive for the deposit and dissemination of scientific research documents, whether they are published or not. The documents may come from teaching and research institutions in France or abroad, or from public or private research centers.
L'archive ouverte pluridisciplinaire HAL, est destinée au dépôt et à la diffusion de documents scientifiques de niveau recherche, publiés ou non, émanant des établissements d'enseignement et de recherche français ou étrangers, des laboratoires publics ou privés. 
Microporous electrostrictive materials for vibrational energy harvesting

\author{
Mickaël Pruvost ${ }^{\mathrm{a}}$, Wilbert J. Smit ${ }^{\mathrm{a}}$, Cécile Monteux ${ }^{\mathrm{a}}$, Philippe Poulin ${ }^{\mathrm{b}}$, Annie Colin ${ }^{\mathrm{a}, \mathrm{b} *}$ \\ a ESPCI Paris, PSL Research University, CNRS, Laboratoire Sciences et Ingénierie de la \\ Matière Molle, UMR 7615, 10 rue Vauquelin, 75231 Paris cedex 05, France \\ $b$ Centre de Recherche Paul Pascal, CNRS, Université de Bordeaux, 115 Avenue Schweitzer, \\ 33600 Pessac, France
}

*Corresponding author : annie.colin@espci.fr

\begin{abstract}
We present electrostrictive materials with excellent properties for vibrational energy harvesting applications. The developed materials consist of a porous carbon black composite, which is processed using water-in-oil emulsions. In combination with an insulating layer, the investigated structures exhibit a high effective relative dielectric permittivity (up to 182 at $100 \mathrm{~Hz}$ ) with very low effective conductivity (down to $2.5310^{-8} \mathrm{~S} \mathrm{~m}^{-1}$ ). They can generate electrical energy in response to mechanical vibrations with a power density of $0.38 \mathrm{~W} \mathrm{~m}^{-3}$ under an applied bias electric field of $32 \mathrm{~V}$. They display figures or merit for energy harvesting applications well above reference polymer materials in the field, including fluorinated co- and ter-polymers synthetized by heavy chemical processes. The production process of the present materials is based on non hazardous and low-cost chemicals. The soft dielectric materials are highly flexible (Young's modulus of $\sim 1 \mathrm{MPa}$ ) making them also suited for highly sensitive capacitive sensors.
\end{abstract}

Keywords: polymers, electrostriction, vibrational energy harvesting, electrostrictive material, capacitive sensors

\title{
1. Introduction:
}

The Internet of Things (IoT) is a new and fast growing concept which aims at connecting everyday physical objects into the Internet without any human interaction $[1,2]$. The IoT requires the usage of numerous wireless sensors. Providing the electrical energy to run these wireless sensors is of major concern. Use of batteries with limited lifetime is a severe constraint. To overcome this problem, several environmental energy sources such as thermal, solar, salinity gradients, vibrations can be used to fulfil energy demands [3-5]. It could thus make sensors, MEMS, communicating devices, etc. self-sufficient in energy supply and as-such highly increase their life time. A promising method to resolve this challenge is to harvest mechanical energy from the ambient environment and convert it into electrical power. An apparently convenient method consists in using piezoelectric materials. The most efficient piezoelectric ceramics can produce up to $0.2-30 \mathrm{~mW} \mathrm{~cm}^{-1}$ [6-9]. However, these materials are stiff and brittle which limits 
their implementation in embedded applications [10]. Triboelectric nanogenerators have recently demonstrated volume power densities up to $0.49 \mathrm{~W} \mathrm{~cm}^{-3}$ [11,12]. However, their performances are largely affected by the environment such as humidity [12]. Moreover, their low durability as a result of two materials in physical friction is still a limiting factor [11]. A more recent approach consists in using electrostrictive polymers to convert energy from mechanical vibrations [13-19]. These polymers have high dielectric permittivity and are used as the dielectric layer inside variable capacitors. Capacitance variations in response to mechanical variations are enhanced by changes of dielectric permittivity of the electrostrictive polymers. This principle can be used to convert mechanical energy of vibrations into electrical energy. The interest in electrostrictive polymers is rapidly growing because polymer-based high-permittivity materials are flexible, low cost, and easily tunable, which makes them good candidates for IoT applications. A recent approach for obtaining high permittivity electrostrictive polymers is the use of polymers loaded with conductive nanoparticles [15-19]. These composite materials exhibit a large increase of dielectric permittivity in the vicinity of their percolation threshold but suffer from a weakness due to the substantial increase of losses near and above percolation $[19,20]$.

To overcome these limitations, we design a new bilayer structure based on a microporous dielectric layer associated with a thin insulating layer of polydimethyl siloxane (PDMS). Porous flexible materials of which the internal pores are covered with carbon black particles are prepared using a water-in-oil emulsion. This new composite possesses a high dielectric permittivity along with a very low conductivity. We show that the fabrication of the microporous dielectric layer using a water-in-oil emulsion provides a fine control of the location of the conductive inclusions. The latter remain confined in the emulsions droplets. The micropores significantly deform upon compression resulting in a large change of the dielectric permittivity [21]. As such, the porous dielectric material can be used in a variable-capacitance engine to harvest mechanical energy [22].The energy harvesting performance can be improved by combining the porous dielectric with a thin insulating layer of PDMS. This reduces the conductivity of the system, and allows an increase of the filler concentration above the percolation threshold in the high permittivity layer. The electrostrictive properties of the material can be finely tuned by varying the concentration of conductive particles. The presented bilayer structures exhibit high effective relative dielectric permittivity (up to 182 at $100 \mathrm{~Hz}$ ) with a very low effective conductivity (down to $2.53 \cdot 10^{-8} \mathrm{~S} \mathrm{~m}^{-}$ ${ }^{1}$ ). They can generate electrical energy in response to mechanical vibrations with a power density 
of $0.4 \mu \mathrm{W} \mathrm{cm} \mathrm{cm}^{-3}$. The efficiency of electrostrictive materials for actual energy harvesting applications can be assessed by their so-called Figure of Merit (FoM). FoM of the present materials exceeds the previous-best electrostrictive materials based on mixtures of fluorinated terpolymers and carbon black particles by a factor 1.7, and by almost four orders magnitude more conventional composites made of polyurethane and carbon black particles. They have also a low cost considering the components they are made of. In addition, considering their large flexibility (Young's modulus of about 1MPa) and sensitivity of dielectric properties, the presented materials are well suited for capacitive sensors with high sensitivity.

\section{Experimental details:}

\subsection{Material synthesis:}

The emulsion consists of an aqueous solution of carbon black droplets dispersed in a matrix of PDMS and a curing agent. We used the following procedure (FIG. 1). The water phase is prepared by mixing $5 \mathrm{~g}$ of arabic gum (Sigma Aldrich) and $95 \mathrm{~g}$ of deionized water. The desired concentration of carbon black powder (Alfa Aesar) is added and the mixture is tip sonicated during 1 hour at $400 \mathrm{~W}$ to homogeneously disperse the carbon black (CB) particles [20]. The oil phase is prepared by mixing PDMS (Dow Corning), the curing agent (Dow Corning, 10\% in weight with respect to the PDMS phase), and the surfactant lauryl PEG-8 dimethicone (Silube J208-812, Siltech, $5.0 \mathrm{wt} \%$ of the final mixture). The emulsion is prepared by progressively adding the carbon black solution to the oil phase under manual stirring up to a mass ratio of water:oil=1:1. The mean droplet size depends on the viscosity of the continuous phase and ranges between $10-30 \mu \mathrm{m}$. The water-in-oil emulsion loaded with carbon black particles is spread with a stencil that has a depth of $500 \mu \mathrm{m}$ on a plastic surface with a diameter of $24 \mathrm{~mm}$. A second plastic surface is placed on the spread emulsion such that the emulsion remains confined between two flat surfaces. Solid materials are obtained by curing the PDMS polymer without evaporation in a warm water bowl $\left(90^{\circ} \mathrm{C}\right)$ for 4 hours. The relative humidity in these conditions is $100 \%$. Then, the solid material layer is removed from the two plastic surfaces and dried in an oven for 1 hour at $150^{\circ} \mathrm{C}$. As PDMS is permeable to water vapor, droplets from carbon black solution dried and leave a structure with spherical-shaped pores covered by carbon black particles (FIG. 2). 
Curing and drying are critical stages which strongly influence the final morphology of the porous composite.

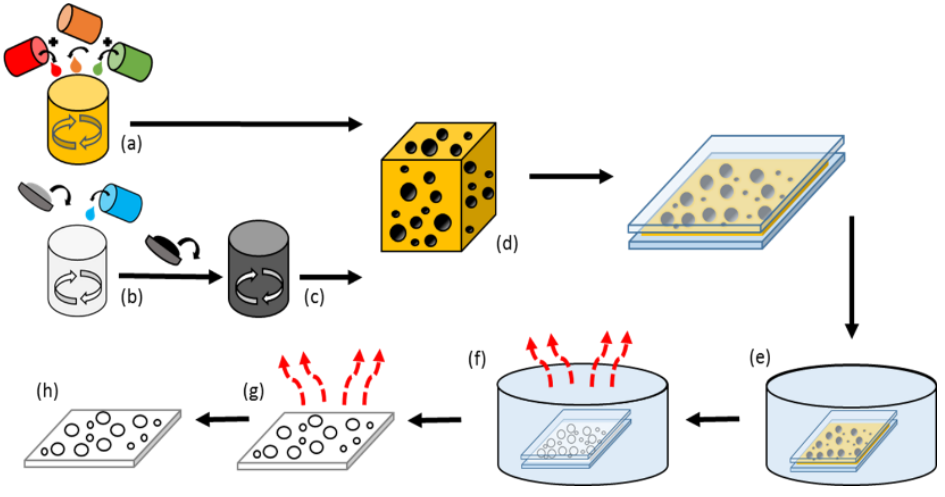

FIG.1: Scheme of the preparation of the microporous materials with pores covered by carbon black particles. (a) Preparation of the oil phase by mixing PDMS, curing agent, and surfactant. (b) Preparation of the water phase by mixing water and arabic gum. (c) Dispersion of black carbon powder in water phase. (d) Dispersion of water phase inside the oil phase. (e) Spreading of the emulsion between two surfaces and immersion in a water bowl. (f) The PDMS in the emulsion is cured by increasing the temperature of water. (g) Evaporation of the water by increasing the temperature outside the water bath. (h) Finally, a porous and dried composite is obtained.

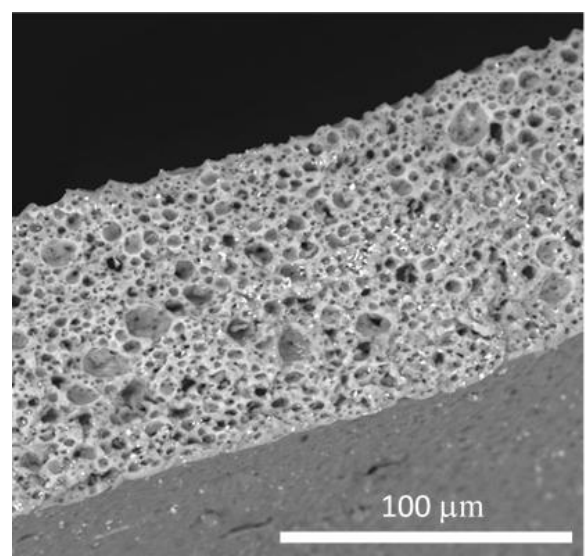

FIG.2: Electron microscope picture of a cross-cut of a porous porous carbon black composite.

\section{Isolating layer deposition:}

To decrease the dielectric losses, the microporous materials are combined with a thin insulating layer of PDMS. The insulating layer is spin coated on conductive aluminum substrates with a diameter of $24 \mathrm{~mm}$. For the spin-coating, $3 \mathrm{~g}$ of PDMS with a curing agent (10\% in weight with respect to the PDMS phase) is deposited at rest on an aluminum substrate. Then a spin-coater is used at $8000 \mathrm{rpm}$ for $60 \mathrm{~s}$ at an acceleration of $1000 \mathrm{rpm} \mathrm{s}^{-1}$ to obtain a thickness of the PDMS layer of $5 \mu \mathrm{m}$. The aluminum substrate covered by the PDMS layer is then soft baked at $100{ }^{\circ} \mathrm{C}$ for 30 minutes to cure the PDMS. The thickness of the layer is measured by a mechanical profilometer (Dektak 150) after scratching the surface. 


\subsection{Determination of the dielectric properties}

We measure the electrical conductivity and dielectric permittivity of the samples between two metallic disc electrodes in the frequency range of $10-10^{6} \mathrm{~Hz}$ under an applied voltage of $1 \mathrm{~V}$ using an impedance analyzer (Bio-Logic Impedance Analyser, MTZ-35). A calibration procedure removing the contribution of the polarization of the electrodes is used to determine the dielectric permittivity and the conductivity of the sample as a function of frequency [16]. All the experiments are performed at room temperature.

\subsection{Determination of the electromechanical performances}

To determine the electrostrictive properties of the composites we use the experimental setup illustrated in FIG. 3, which consists of a piezoelectric actuator (Physik Instrumente P-842.30) driven by a function generator (Keysight 33611A Waveform Generator) that applies a sinusoidal mechanical strain at a frequency of $100 \mathrm{~Hz}$. The sample is placed between two electrodes: the top electrode is connected to the piezo actuator and moves at the same frequency as the actuator, the bottom electrode is fixed to the sample holder and does not move. As such, a modulated strain is applied along the thickness of the material. The strain is controlled by a computer linked to a function generator which controls the stroke of the actuator. An electric field $E_{d c}$ is applied to the composite along the height (3-direction in FIG. 3) by a DC generator. To measure the current and generated power, a load resistance $(R)$ is connected in series with the sample. The voltage $U_{R}$ over the resistance is recorded by an oscilloscope (Keysight InfiniiVision $1000 \mathrm{X}$ ) and the current is determined using the Ohm's law $\left(I=U_{R} / R_{l}\right)$.

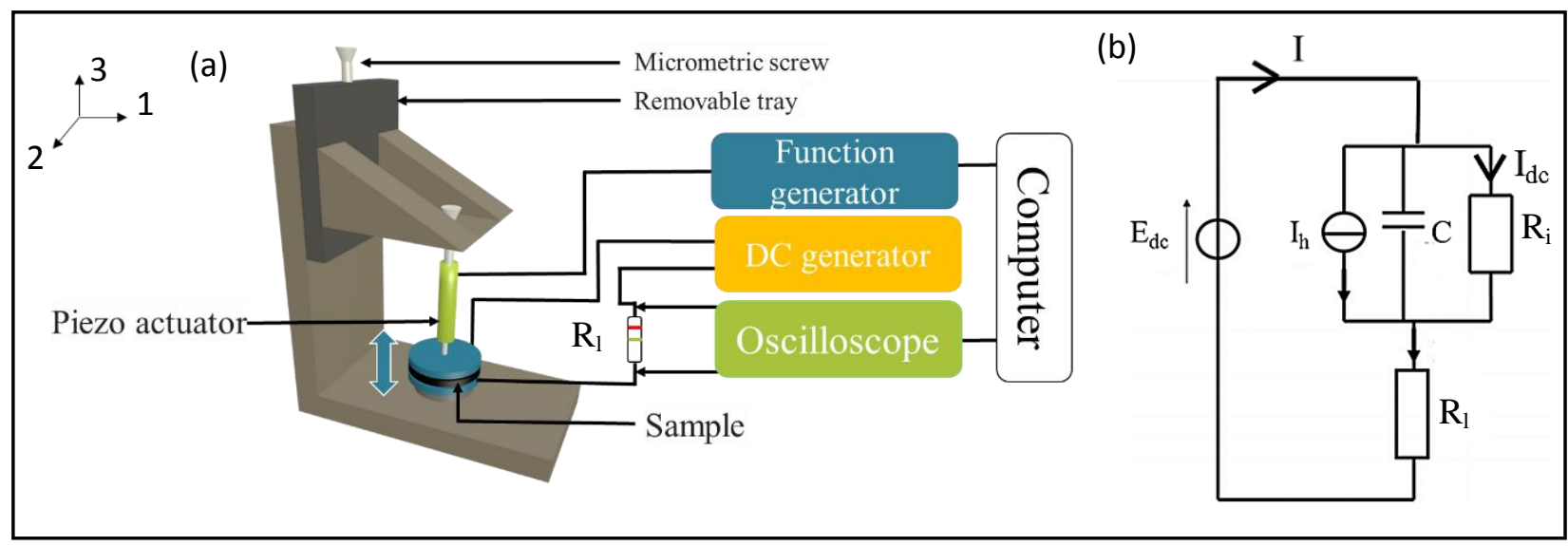

FIG.3: Schematic of the experimental setup (a) and the equivalent electric circuit (b). 
The Young's modulus of the composites is measured using a tensile tester (Instron Model 4505). The initial dimensions (width and thickness) are measured before mounting the sample between the fixed and moving jaws. The force-displacement responses are acquired for each sample at a displacement rate of $0.067 \mathrm{~mm} \mathrm{~s}^{-1}$. The tests ended when the specimens fractured. The Young's modulus is determined by measuring the slope of stress-strain curves in the elastic domain.

The composite material is modeled by a parallel $R C$ circuit (see FIG. 3b). The observed current is composed of two parts: a leakage current $I_{d c}$ and a harvested current $I_{h}$ induced by compression-decompression cycles of the composite. According to the model developed by Guyomar et al $[23,24]$, for a sinusoidal strain the current $I_{h}$ through a load resistance $R_{l}$ is given by (see supporting information)

$I_{h}(t)=\frac{2 M_{33}{ }^{*} Y E_{d c} \omega A S}{1+\left(\frac{\omega A R_{l} \varepsilon \varepsilon_{0}}{d}\right)^{2}}\left[\frac{\omega A R_{l} \varepsilon \varepsilon_{0}}{d} \sin (\omega t)+\cos (\omega t)\right]$

Here, $A$ is the area of the sample, $Y$ is the Young's modulus, $M_{33}{ }^{*}$ is the apparent electrostrictive coefficient [15], $E_{d c}$ is the applied bias electric field $\left(E_{d c}=32 \mathrm{~V}\right), S$ is the sinusoidal strain amplitude equaling the displacement induced by the piezo actuator $(24 \mu \mathrm{m})$ divided by two times the material thickness, $\varepsilon$ is the relative dielectric permittivity, $\varepsilon_{0}$ is the vacuum permittivity, $d$ is the material thickness, and $\omega$ is the angular frequency of the applied strain. Hence, a linear relation between $I_{h}$ and $E_{d c}$ is obtained for a constant strain, frequency, and load resistance. As a result, the product $M_{33}{ }^{*} Y$ can be easily estimated from the slope of the $I-E_{d c}$ curves. The apparent electrostrictive coefficient $M_{33}{ }^{*}$ is calculated by division of the Young's modulus $Y$.

\section{Results:}

\subsection{Polymer-carbon black composites:}

The dielectric properties of the polymer/carbon black composite, without insulating layer, are first studied at rest. The load fraction of carbon black particles inside composites is varied while keeping the oil:water ratio constant. FIG. 4 shows the electrical conductivity, dielectric permittivity, and loss tangent $\left(\tan (\delta)=\frac{\sigma}{\varepsilon_{0} \varepsilon \omega}\right.$, with $\sigma$ the electrical conductivity) as a function of the frequency for different carbon black particle concentrations. 

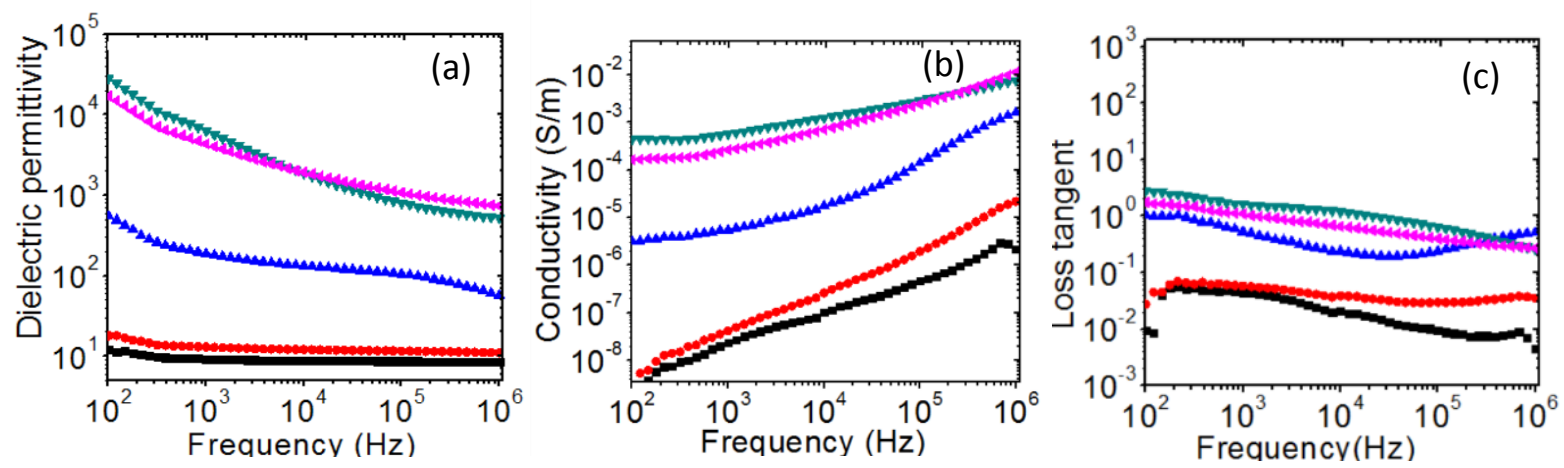

FIG. 4 : a) Evolution of the dielectric permittivity (a), conductivity (b) and loss tangent (c) as a function of the frequency for various $C B$ particle concentrations. $3.0 \mathrm{wt} \%$ (black squares), $4.0 \mathrm{wt} \%$ (red circles), $6.0 \mathrm{wt} \%$ (purple top triangles), $8.0 \mathrm{wt} \%$ (green down triangles) , 10 $w t \%$ (pink left triangles)Phase fractions are fixed: oil:water $=1: 1$

Both conductivity and dielectric permittivity increase as a function of $\mathrm{CB}$ concentration. The percolation threshold is reached for a weight fraction of $6 \%$. Below the percolation threshold, the conductivity is frequency dependent and arises from dielectric losses due to bound charges. In the meantime, the dielectric permittivity remains constant for the full range of investigated frequencies. Above the percolation threshold, and at low frequencies, the conductivity is constant because essentially due to the transport of free charges brought by the carbon black particles. By contrast, the dielectric permittivity presents frequency dependence and is enhanced by the interfacial polarization of the carbon black particles. In the high filler concentration regimes, the values of conductivity (above $10^{-6} \mathrm{~S} \mathrm{~m}^{-1}$ ) make the materials unsuitable for vibrational energy harvesting.

\subsection{Bilayer polymer-carbon black composites:}

The high conductivity (free charges carriers and dielectric loss) of the carbon black composites is a drawback for energy harvesting applications [24]. Indeed, such applications do not allow any leakage for yielding a positive energy conversion. In this paper, we propose an original approach for keeping high values of effective dielectric permittivity and low values of conductivity by associating a thin insulating layer to the high permittivity layer. This layer has low dielectric losses and no free charge carriers. It acts as a blocking layer and prevents leaks of the capacitors (FIG. 5). 

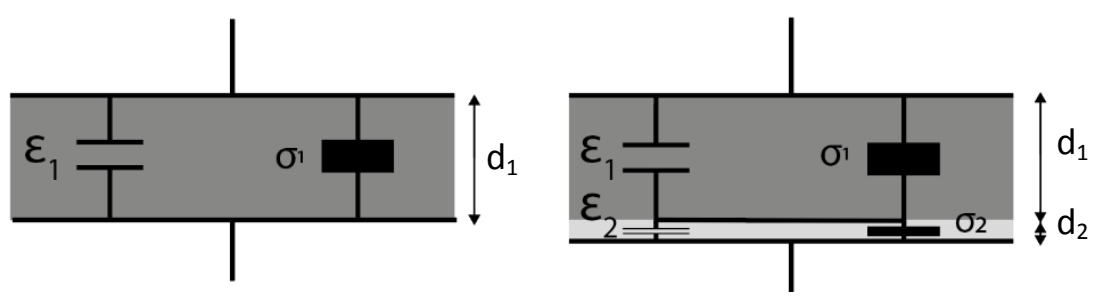

FIG. 5: Equivalent electric circuits for the porous black carbon (a) and the bilayer (b) composite. $\varepsilon_{i}, \sigma_{i}$ and $d_{i}$ are the dielectric permittivity, the conductivity, and the thickness of the layer $i$, respectively.

The electrical equivalent circuit for a bilayer material is a junction of two RC circuits in series. The expressions for the effective dielectric properties of the bilayer structure are given in the supporting information. The effective conductivity and dielectric constant of the bilayer structure depend on the thicknesses and dielectric properties of both layers. The blocking layer 2 is a cured PDMS layer $\left(\varepsilon_{P D M S}=\varepsilon_{2}=2\right.$ and $\sigma_{2}=10^{-13} \mathrm{~S} \mathrm{~m}^{-1}$ in the range $10-10^{6} \mathrm{~Hz}$ at $\left.25^{\circ} \mathrm{C}\right)$ of thickness $5 \mu \mathrm{m}$. The high permittivity layer 1 is the porous carbon black material layer $\left(\varepsilon_{1} \gg\right.$ $\varepsilon_{2}$ and $\sigma_{1} \ll \sigma_{2}$ ) of variable thickness depending on carbon black concentration (see Table 1).

Table 1: Properties of the carbon black $(\mathrm{CB})$ composites.

\begin{tabular}{ccc}
$\% \mathrm{CB}$ & $\mathrm{CB}\left(\mathrm{g} \mathrm{L}^{-1}\right)$ & Thickness $(\mu \mathrm{m})$ \\
\hline \hline 3 & 31 & 500 \\
\hline 4 & 42 & 510 \\
\hline 6 & 64 & 510 \\
\hline 8 & 87 & 750 \\
\hline 10 & 110 & 800 \\
\hline
\end{tabular}
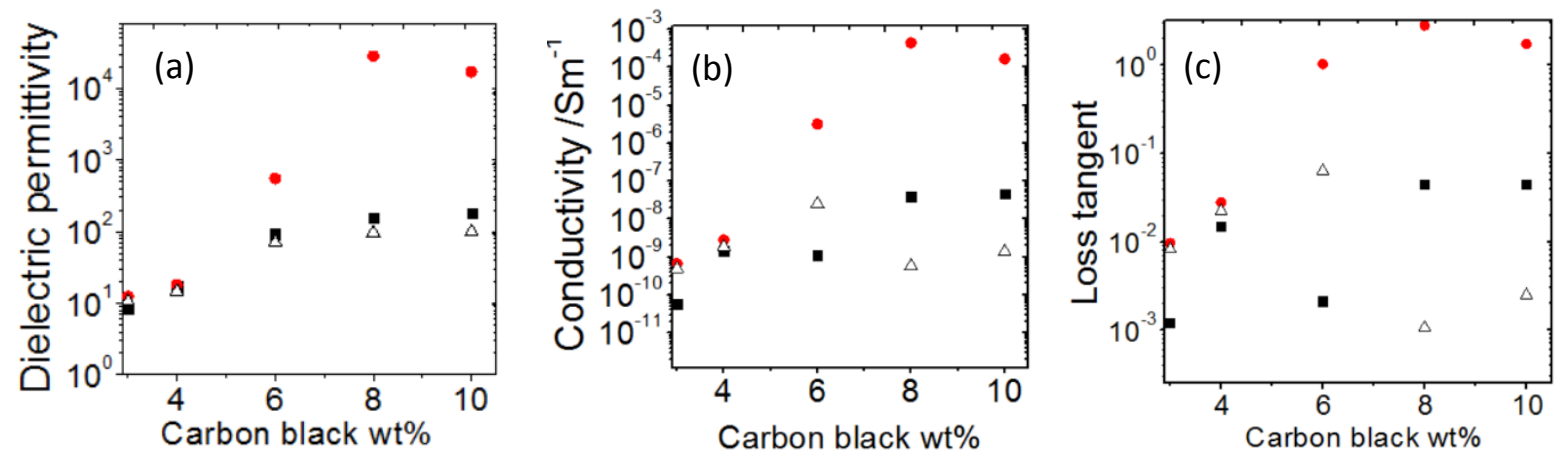

FIG. 6: Effective dielectric properties at $100 \mathrm{~Hz}$ of the porous carbon black layer (red circles), bilayer polymer - carbon black composites (black squares), and theoretical predictions of the latter (open black triangles), as a function of carbon black concentration. 
For comparison, we investigate the dielectric properties of the electrostrictive composites at rest with and without insulating layer. FIG. 6 shows the relative dielectric permittivity, electrical conductivity, and loss tangent at $100 \mathrm{~Hz}$ as a function of the carbon black concentration. First, we consider the electrostrictive composites without insulating layer. At carbon black concentrations below the percolation threshold $(\sim 5 \mathrm{wt} \%)$, the dielectric permittivity has a nearly constant value of $\sim 10$ with an electric conductivity of $\sim 1 \mathrm{nS} \mathrm{m}^{-1}$. Above the percolation threshold, the dielectric permittivity rapidly increases to 550 at $6 \mathrm{wt} \%$. However, the electrical conductivity also increases by a factor of $10^{3}$, leading to a loss tangent above 1 . A further addition of conductive carbon black particles enhances the interfacial polarization, which leads to an increase in permittivity but also leads to further increase of the electrical conductivity to $0.6 \mathrm{mS} \mathrm{m}^{-1}$ at $\sim 8 \mathrm{wt} \%$. Such a high conductivity does not allow the efficient use of the materials as a dielectric layer in variable capacitors.

The effective dielectric properties of the electrostrictive composites with an insulating layer are very different at carbon black concentrations above the percolation threshold. The PDMS layer limits the electric conductivity below $1-10 \mathrm{nS} \mathrm{m}^{-1}$. The insulating layer also leads to a decrease of the effective dielectric permittivity to 157 and 182 for the 8 and $10 \mathrm{wt} \%$ black carbon weight fractions, respectively. However, the obtained dielectric loss tangents are very low with $1.5 \cdot 10^{-2}$ and $2.5 \cdot 10^{-2}$ for the 8 and $10 \mathrm{wt} \%$ carbon black systems, respectively, making them well suited for energy harvesting applications. Theoretical calculations of the conductivity (see supporting information) predict values slightly lower than the ones found experimentally, probably as a result of small holes and defects in the insulating PDMS layer.

\subsection{Electromechanical performances and power harvested estimation:}

We now investigate the performance of the bilayer composites for energy harvesting purposes. First, the electromechanical performances are assessed by calculating the figure of merit (FoM) proposed by Guyomar et al. [23,24]:

$\mathrm{FoM}=\left(\frac{2 \pi}{\varepsilon_{r}}\right)\left(M_{33}{ }^{*} Y\right)^{2} \quad\left(\mathrm{~J} \mathrm{~m}^{-1} \mathrm{~V}^{-2} \mathrm{cycle}^{-1}\right)$ 
The FoM depends only on materials properties $\left(M_{33}{ }^{*}, Y, \varepsilon_{r}\right)$ and aims at comparing electromechanical performances of materials without any external parameters such as geometry and measurement details. Three compositions $(6,8$, and $10 \mathrm{wt} \%)$ are used for performances comparisons (Table 2). Properties of the materials are reported in Table 2. FoM, $M_{33}{ }^{*}$ and $Y$ are measured using the methods described in the experimental section.

Table 2: Materials properties

\begin{tabular}{cccccccc}
$\% \mathrm{CB}$ & $\begin{array}{c}\text { Oil } \\
\text { fraction }\end{array}$ & $\mathrm{CB}\left(\mathrm{g} \mathrm{L}^{-1}\right)$ & $\begin{array}{c}\text { Thickness } \\
(\mu \mathrm{m})\end{array}$ & $\begin{array}{c}\text { Surface } \\
\left(\mathrm{m}^{2}\right)\end{array}$ & $M^{*} Y$ & $\begin{array}{c}Y \\
(\mathrm{MPa})\end{array}$ & $\begin{array}{c}M_{33}{ }^{*} \\
\left(\mathrm{~m}^{2} \mathrm{~V}^{-2}\right)\end{array}$ \\
\hline \hline 6.0 & 0.5 & $6.4 \cdot 10^{1}$ & $5,1 \cdot 10^{2}$ & $1.1 \cdot 10^{-3}$ & $2.2 \cdot 10^{-10}$ & 1,38 & $\begin{array}{c}1,60 \cdot 10^{-} \\
16\end{array}$ \\
\hline \hline 10.0 & 0.5 & $8,7 \cdot 10^{1}$ & $7.5 \cdot 10^{2}$ & $6.2 \cdot 10^{-4}$ & $1.5 \cdot 10^{-9}$ & 1,40 & $\begin{array}{c}1,07 \cdot 10^{-} \\
15\end{array}$ \\
\hline 0.5 & $1.1 \cdot 10^{2}$ & $8.0 \cdot 10^{2}$ & $1.0 \cdot 10^{-3}$ & $3.6 \cdot 10^{-9}$ & 1,61 & $\begin{array}{c}8,46 \cdot 10^{-} \\
15\end{array}$
\end{tabular}

\subsection{Determination of the harvested power:}

Next, we examine the power generation properties of the bilayer composites by discharge over a load resistance $R_{l}$. As shown in FIG. 8, the voltage drop over the resistance $U_{r}$ and the instantaneous harvested power are monitored as a function of time. The average harvested power per cycle $\bar{P}$ is calculated using

$\bar{P}=\frac{1}{T} \int_{0}^{T} P(t) \mathrm{d} t=\frac{1}{R_{l} T} \int_{0}^{T} U_{r}(t)^{2} \mathrm{~d} t$,

where $T$ is the period of the cycle.

(a)

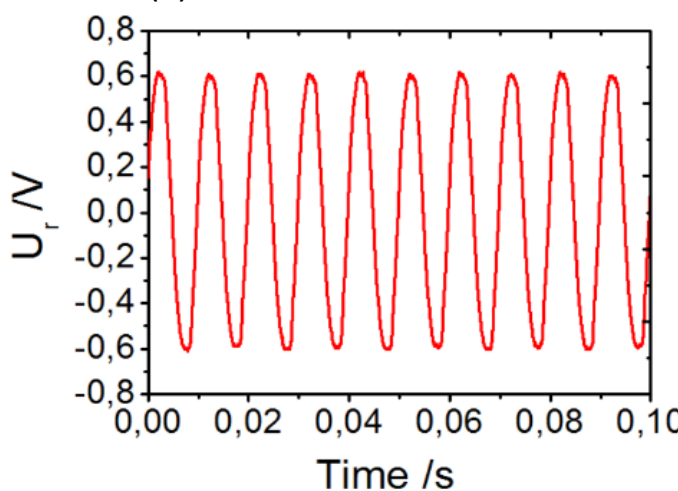

(b)

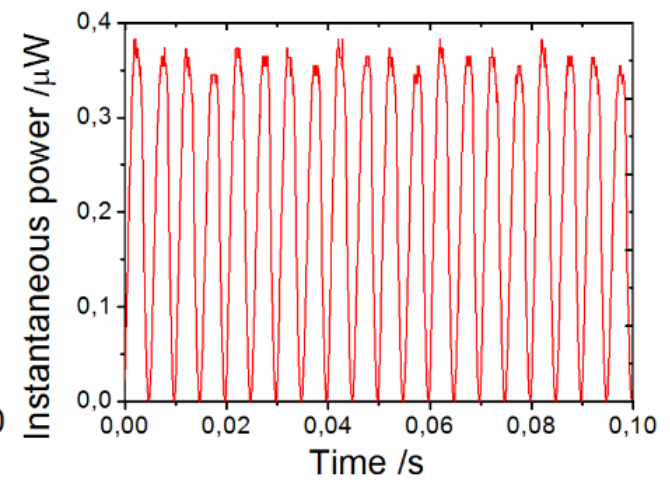

FIG. 8: The voltage difference (a) and instantaneous power (b) across a load resistance of $1 \mathrm{M} \Omega$ for a $100 \mathrm{~Hz}$ sinusoidal strain. The applied bias voltage over 10 the bilayer composite with 8 wt\% carbon black is $32 \mathrm{~V}$. 
The theoretical harvested power is given by (see supporting information)

$\overline{P_{\mathrm{th}}}=\frac{R_{l}}{2} \frac{\left(2 M_{33}{ }^{*} Y\right)^{2} E_{d c^{2} \omega^{2} A^{2} S^{2}}}{1+\left(\frac{R_{l} A \varepsilon_{0} \varepsilon \omega}{d}\right)^{2}}$

where $\omega=2 \pi / T$.

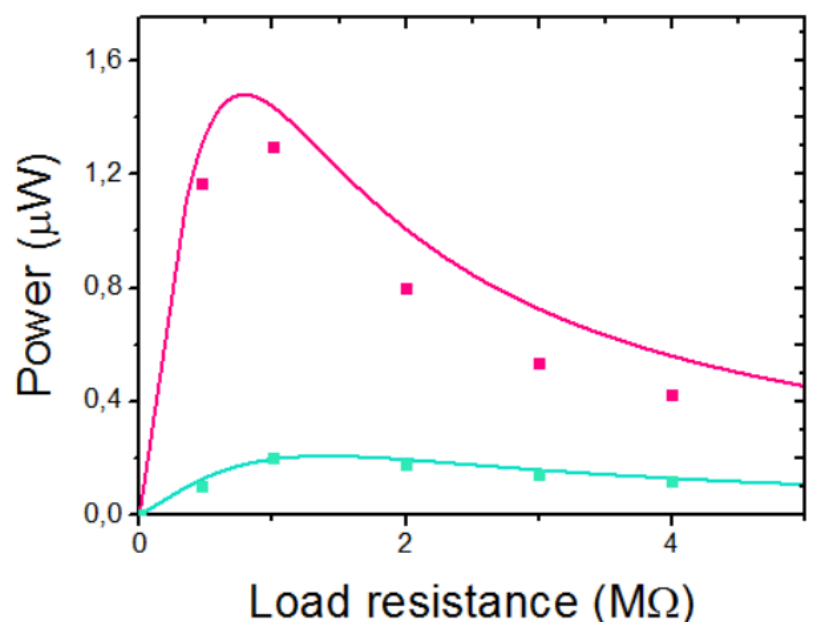

FIG. 9: Harvested power of the composites with $8 C B$ wt\% (green) and $10 C B$ wt\% (pink) with insulating layer as a function of the load resistance. Squares are experimental data and lines are theoretical predictions using EQ. 7. The strain frequency is $100 \mathrm{~Hz}$ and the applied voltage over the composite $V_{d c}=32 \mathrm{~V}$.

FIG. 9 shows the value of the theoretical and experimental power harvestable as a function of the load resistance. Best values are obtaining for a load resistance of about $1 \mathrm{M} \Omega$ for both materials, which corresponds to the resistance with optimal power production $\left(R_{l, \max }=\frac{d}{A \varepsilon \varepsilon_{0} \omega}\right)$. Good agreement is observed between the experimental data and theoretical powers. In addition, the increase in FoM by a factor 5 between $8 \mathrm{CB}$ wt $\%$ and $10 \mathrm{CB}$ wt $\%$ leads to an increase in harvested power by a factor 6 .

\subsection{Efficiency:}


To get a positive energy balance, the dissipated power must be smaller than the harvested power. The dissipated power for maintaining a polarization of the composite is given by [24]:

$P_{\text {loss }}=R_{l}\left(\frac{V_{d c}}{R_{i}+R_{l}}\right)^{2}$

where $R_{i}$ is the internal leak resistance, $R_{l}$ is the load resistance values, and $V_{d c}$ is the applied voltage difference. The internal leak resistance is calculated by $R_{i}=\frac{d}{\sigma A}$ with the geometrical data from Table 2. The electrical energy gain is calculated by considering the ratio of power harvestable to electrical polarization losses for a bias voltage of $V_{d c}=32 \mathrm{~V}$ and a load resistance of $R_{l}=1 \mathrm{M} \Omega$.

Table 3: Efficiency of the bilayer composites for different carbon black concentrations.

\begin{tabular}{ccccc} 
\% CB & Harvested power $(\mu \mathrm{W})$ & Power loss $(\mu \mathrm{W})$ & $\begin{array}{c}\text { Net power } \\
\text { production } \\
(\mu \mathrm{W})\end{array}$ & $\begin{array}{c}\text { Electrical gain } \\
\text { factor }\end{array}$ \\
\hline \hline 8.00 & 0.20 & 0.12 & 0.08 & 1.74 \\
\hline 10.0 & 1.3 & 1.0 & 0.3 & 1.31
\end{tabular}

\section{Discussions:}

The use of an insulating layer limits the electric conductivity below $1-10 \mathrm{nS} \mathrm{m}^{-1}$ and allows us to use our materials for energy harvesting. Data given in Table 3 show that the energy balance is largely positive for both materials. The power produced by the mechanical-electrical conversion for the $8 \mathrm{wt} \%$ composite is 1.74 times higher than the power dissipated to polarize it and the net power harvestable is around $0.17 \mathrm{~W} \mathrm{~m}^{-3}$. We can see that increasing the $\mathrm{CB}$ wt\% enables to increase the net harvested power by almost a factor 4 , i.e. up to $0.38 \mathrm{~W} \mathrm{~m}^{-3}$. This is a consequence of the increase of permittivity of the material at $10 \mathrm{wt} \%$. But at the same time, the conductivity of the $10 \mathrm{wt} \%$ composite is 1.9 higher than that of the $8 \mathrm{wt} \%$ composite which has a conductivity of $1.30 \cdot 10^{-8} \mathrm{~S} \mathrm{~m}^{-1}$; leading to a net decrease in the energy balance, but to an increased overall power production. These excellent performances can be explained by the porous structure of the materials and insulating properties of the thin polymer layer. As a result, the electric field required to polarize the material is decreased, which is crucial for vibrational energy harvesting applications. 
FIG. 7 presents the FoM of materials from the literature, and of composites developed in this work. The values of the FOM are enhanced by increasing the filler concentration. For 10 wt $\%$ $\mathrm{CB}$, the FoM is equal to $5.06 \cdot 10^{-8} \mathrm{~J} \mathrm{~m}^{-1} \mathrm{~V}^{-2}$ cycle ${ }^{-1}$. This result exceeds the previous-best electrostrictive materials based on a mixture of Polyvinylidene fluoride (PVDF) terpolymers and carbon black by a factor 1.7. In addition, it outperforms in term of process and material cost and toxicity [21-22]. Increasing the FoM is a key point for increasing the power harvestable as we show below. Besides, their low Young's modulus compared to PVDF polymers ( GPa) could enlarge the scope of applications using low stress vibrations.

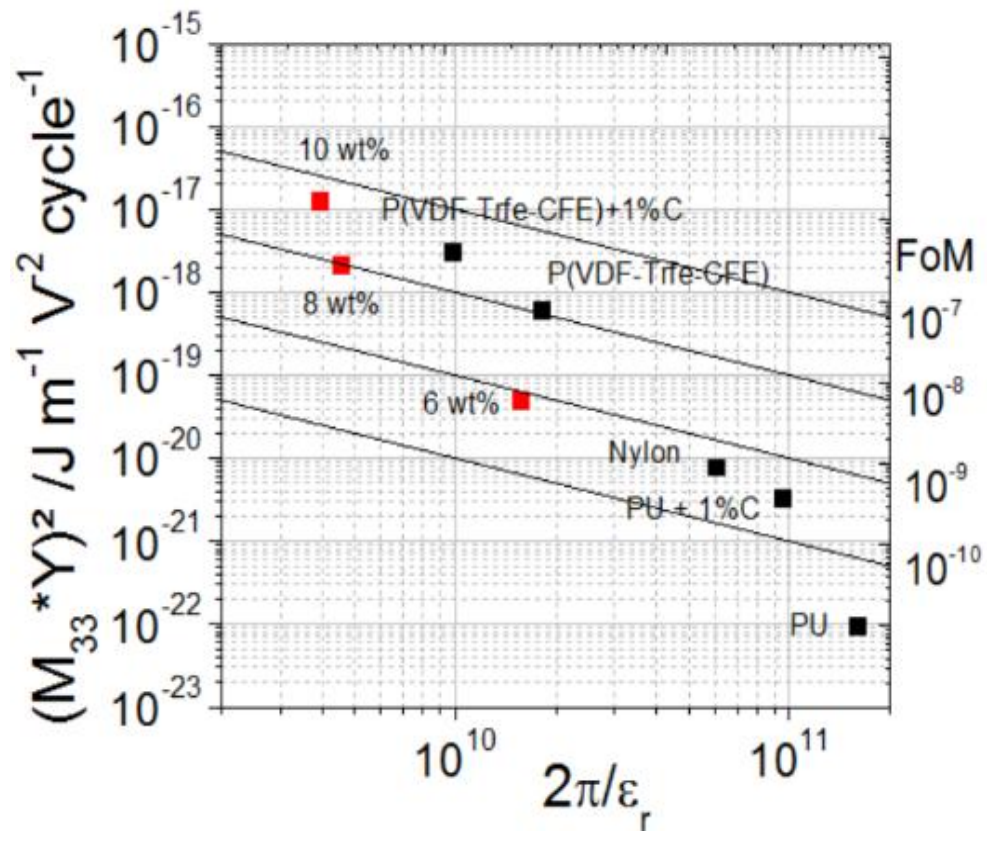

FIG. 7: Comparison of the electromechanical performances at $100 \mathrm{~Hz}$ using the figure of merit (FoM) proposed by Guyomar et al. [23,24]. Thick lines have the same FOM. Black spots refer to materials from the literature [23,24].

\section{Conclusions:}

We presented microporous electrostrictive composites with improved properties for vibrational energy harvesting. These systems are made of carbon black particles dispersed in a porous PDMS matrix. An inverted emulsion templating approach provides a fine control of the location of the particles which is a key factor to achieve high permittivity and low losses. A thin insulating layer was added to limit further the losses. The resulting structure possesses a very large effective 
relative dielectric permittivity of 182 at $100 \mathrm{~Hz}$ in combination with a very low loss tangent of $2.5 \cdot 10^{-2}$. We succeeded in evaluating the energy scavenging abilities of these composites. More than $300 \mathrm{nW}$ has been harvested with the $10 \mathrm{wt} \%$ carbon black composites which leads to a power density of $0.4 \mu \mathrm{W} \mathrm{cm}$. The present materials exhibits a figure of merit as high as $5.06 \cdot 10^{-8} \mathrm{~J} \mathrm{~m}^{-1} \mathrm{~V}^{-2}$ cycle $^{-1}$ which is 1.7 times higher than the best reference fluorinated polymers and 3350 times higher than pure polyurethane [21]. The low cost process and low toxicity make the carbon black bilayer composites very promising materials for vibrational energy harvesting. The power harvested with our materials is good enough to provide a significant boost to the battery life of ultra-low power devices. For example, the Phoenix processor [25,26] has a sleep power consumption of only $30 \mathrm{pW}$ and $200 \mathrm{nW}$ in active mode which makes our materials suitable for this application. In addition, these carbon black bilayer composites, with excellent dielectric properties, could find potential applications as low cost flexible capacitors sensors in the printed electronics industry.

Acknowledgements: We acknowledge financial support from the following organisms and projects: Université de recherche Paris Sciences et Lettres, the Université Pierre et Marie Curie, ANR Project ANR-12-NANO-0002 ELENA, and ANR in the Cluster of Excellence AMADEUS. 


\section{Supporting information}

\subsection{Effective dielectric properties of a stacked bilayer}

The effective relative dielectric permittivity $\varepsilon$ and conductance $\sigma$ of two stacked dielectric layers are calculated by [27]

$\varepsilon=\varepsilon_{h}+\frac{\varepsilon_{l}-\varepsilon_{h}}{1-\omega^{2} \tau^{2}}$

$\sigma=\sigma_{l}+\varepsilon_{0}\left(\varepsilon_{l}-\varepsilon_{h}\right) \frac{\omega^{2} \tau}{1-\omega^{2} \tau^{2}}$

with

$\varepsilon_{h}=\frac{\varepsilon_{1} \varepsilon_{2} d}{\varepsilon_{1} d_{2}+\varepsilon_{2} d_{1}}$

$\varepsilon_{l}=\frac{\varepsilon_{1} \sigma_{2}^{2} d_{1} d+\varepsilon_{2} \sigma_{1}^{2} d_{2} d}{\left(\sigma_{1} d_{2}+\sigma_{2} d_{1}\right)^{2}}$

$\tau=\varepsilon_{0} \frac{\varepsilon_{1} d_{2}+\varepsilon_{2} d_{1}}{\sigma_{1} d_{2}+\sigma_{2} d_{1}}$

$\sigma_{l}=\frac{\sigma_{1} \sigma_{2} d}{\sigma_{1} d_{2}+\sigma_{2} d_{1}}$.

Here, $d_{1}$ is the thickness of layer $1, d_{2}=d-d_{1}$ is the thickness of layer $2 . \varepsilon_{i}$ and $\sigma_{i}$ are the dielectric constant and conductivity of layer $i$, respectively, and $\varepsilon_{0}$ is the vacuum permittivity.

\subsection{Theoretical derivation of the harvested current}

Here, we expose a theoretical derivation of the harvested current for a pure electrostrictive material (i.e. no piezoelectric behaviour). We depart from the differential equation for the harvested current $I_{h}$ as derived by Lallart et al. [21] for a small strain $S$ :

$I_{h}(t)=-\frac{A R_{l} \varepsilon \varepsilon_{0}}{d} \frac{\partial I_{h}(t)}{\partial t}+2 A M_{33}{ }^{*} Y E_{d c} \frac{\partial S(t)}{\partial t}$ 
For convenience, we define $\mathcal{A} \equiv \frac{A R_{l} \varepsilon \varepsilon_{0}}{d}$ and $\mathcal{B} \equiv 2 A M_{33}{ }^{*} Y E_{d c}$. The above equation can then be rewritten as

$\frac{I_{h}(t)}{\mathcal{A}}+\frac{\partial I_{h}(t)}{\partial t}=\frac{\mathcal{B}}{\mathcal{A}} \frac{\partial S(t)}{\partial t}$.

Multiplying both sides by $\mathrm{e}^{\frac{t}{\mathcal{A}}}$ gives

$$
\mathrm{e}^{\frac{t}{\mathcal{A}}}\left(\frac{I_{h}(t)}{\mathcal{A}}+\frac{\partial I_{h}(t)}{\partial t}\right)=\mathrm{e}^{\frac{t}{\mathcal{A}}} \frac{\mathcal{B}}{\mathcal{A}} \frac{\partial S(t)}{\partial t} .
$$

Integrating both sides yields

$$
\begin{gathered}
\mathrm{e}^{\frac{t}{\mathcal{A}}} I_{h}(t)=\mathcal{C}+\frac{\mathcal{B}}{\mathcal{A}} \int_{-\infty}^{t} \mathrm{~d} t^{\prime} \mathrm{e}^{\frac{t^{\prime}}{\mathcal{A}}} \frac{\partial S\left(t^{\prime}\right)}{\partial t^{\prime}} \\
I_{h}(t)=\mathcal{C} \mathrm{e}^{\frac{-t}{\mathcal{A}}}+\frac{\mathcal{B}}{\mathcal{A}} \mathrm{e}^{\frac{-t}{\mathcal{A}}} \int_{-\infty}^{t} \mathrm{~d} t^{\prime} \mathrm{e}^{\frac{t^{\prime}}{\mathcal{A}}} \frac{\partial S\left(t^{\prime}\right)}{\partial t^{\prime}},
\end{gathered}
$$

where $\mathcal{C}$ is an integration constant, which we set to zero as $\lim _{t \rightarrow \pm \infty} I_{h}(t)$ is finite. This leaves us with the following integral:

$$
I_{h}(t)=\frac{\mathcal{B}}{\mathcal{A}} \mathrm{e}^{\frac{-t}{\mathcal{A}}} \int_{-\infty}^{t} \mathrm{~d} t^{\prime} \mathrm{e}^{\frac{t^{\prime}}{\mathcal{A}}} \frac{\partial S\left(t^{\prime}\right)}{\partial t^{\prime}}
$$

For a sinusoidal strain with amplitude $\mathrm{S}$ and angular frequency $\omega$, i.e.

$S(\mathrm{t})=\mathrm{S} \sin (\omega t)$,

the harvested current becomes

$I_{h}(t)=\omega S \frac{\mathcal{B}}{\mathcal{A}} \mathrm{e}^{\frac{-t}{\mathcal{A}}} \int_{-\infty}^{t} \mathrm{~d} t^{\prime} \mathrm{e}^{\frac{t^{\prime}}{\mathcal{A}}} \cos \left(\omega t^{\prime}\right)$

By applying integration by parts twice we get

$I_{h}(t)=\frac{\omega}{1+\omega^{2} \mathcal{A}^{2}} S \mathcal{B}[\omega \mathcal{A} \sin (\omega t)+\cos (\omega t)]$ 
We now insert the definitions for $\mathcal{A}$ and $\mathcal{B}$ and obtain

$$
I_{h}(t)=\frac{2 M_{33}{ }^{*} Y E_{d c} \omega A S}{1+\left(\frac{\omega A R_{l} \varepsilon \varepsilon_{0}}{d}\right)^{2}}\left[\frac{\omega A R_{l} \varepsilon \varepsilon_{0}}{d} \sin (\omega t)+\cos (\omega t)\right] .
$$




\subsection{Derivation of the harvested power}

The average harvested power of a cycle $\bar{P}$ can be obtained by calculating

$$
\bar{P}=\frac{R_{l}}{T} \int_{0}^{T} \mathrm{~d} t I_{h}{ }^{2}(t)
$$

where $T=2 \pi / \omega$ is the period of the cycle.

Inserting the above derived expression for $I_{h}(t)$, we obtain

$$
\begin{gathered}
\bar{P}=\frac{R_{l}}{T}\left[\frac{2 M_{33}{ }^{*} Y E_{d c} \omega A S}{1+\left(\frac{\omega A R_{l} \varepsilon \varepsilon_{0}}{d}\right)^{2}}\right]^{2} \int_{0}^{T} \mathrm{~d} t\left[\frac{\omega A R_{l} \varepsilon \varepsilon_{0}}{d} \sin ^{2}(\omega t)+\cos ^{2}(\omega t)\right] \\
\bar{P}=\frac{R_{l}}{2} \frac{\left(2 M_{33}{ }^{*} Y E_{d c} \omega A S\right)^{2}}{1+\left(\frac{R_{l} \varepsilon \varepsilon_{0} \omega A}{d}\right)^{2}} .
\end{gathered}
$$


References:

[1] J. Gubbi, R. Buyya, S. Marusic, M. Palaniswami, Internet of Things (IoT): A vision, architectural elements, and future directions, Future Gener. Comput. Syst. 29 (2013) 16451660. doi:10.1016/j.future.2013.01.010.

[2] F. Xia, L.T. Yang, L. Wang, A. Vinel, Internet of Things, Int. J. Commun. Syst. 25 (2012) 1101-1102. doi:10.1002/dac.2417.

[3] D. Brogioli, Extracting Renewable Energy from a Salinity Difference Using a Capacitor, Phys. Rev. Lett. 103 (2009). doi:10.1103/PhysRevLett.103.058501.

[4] M. Janssen, A. Härtel, R. van Roij, Boosting Capacitive Blue-Energy and Desalination Devices with Waste Heat, Phys. Rev. Lett. 113 (2014). doi:10.1103/PhysRevLett.113.268501.

[5] N.E. Dutoit, B.L. Wardle, S.-G. Kim, Design considerations for mems-scale piezoelectric mechanical vibration energy harvesters, Integr. Ferroelectr. 71 (2005) 121-160. doi: $10.1080 / 10584580590964574$.

[6] H.A. Sodano, D.J. Inman, G. Park, A Review of Power Harvesting from Vibration Using Piezoelectric Materials, Shock Vib. Dig. 36 (2004) 197-205. doi:10.1177/0583102404043275.

[7] S.R. Anton, H.A. Sodano, A review of power harvesting using piezoelectric materials (2003-2006), Smart Mater. Struct. 16 (2007) R1-R21. doi:10.1088/0964-1726/16/3/R01.

[8] Y. Yan, J.E. Zhou, D. Maurya, Y.U. Wang, S. Priya, Giant piezoelectric voltage coefficient in grain-oriented modified PbTiO3 material, Nat. Commun. 7 (2016) 13089. doi: $10.1038 /$ ncomms 13089.

[9] F.R. Fan, W. Tang, Z.L. Wang, Flexible Nanogenerators for Energy Harvesting and SelfPowered Electronics, Adv. Mater. 28 (2016) 4283-4305. doi:10.1002/adma.201504299.

[10] O. Guillon, F. Thiebau, D. Perreux, Tensile fracture of soft and hard PZT, Int. J. Fract. (2002). doi:https://doi.org/10.1023/A:1022072500963.

[11] Z.L. Wang, Triboelectric nanogenerators as new energy technology and self-powered sensors - Principles, problems and perspectives, Faraday Discuss. 176 (2014) 447-458. doi:10.1039/C4FD00159A.

[12] V. Nguyen, R. Yang, Effect of humidity and pressure on the triboelectric nanogenerator, Nano Energy. 2 (2013) 604-608. doi:10.1016/j.nanoen.2013.07.012.

[13] D. Jaaoh, C. Putson, N. Muensit, Enhanced strain response and energy harvesting capabilities of electrostrictive polyurethane composites filled with conducting polyaniline, Compos. Sci. Technol. 122 (2016) 97-103. doi:10.1016/j.compscitech.2015.11.020.

[14] X. Yin, J.-F. Capsal, D. Guyomar, A comprehensive investigation of poly(vinylidene fluoride-trifluoroethylene-chlorofluoroethylene) terpolymer nanocomposites with carbon black for electrostrictive applications, Appl. Phys. Lett. 104 (2014) 052913. doi:10.1063/1.4864160.

[15] A. Luna, M. Pruvost, J. Yuan, C. Zakri, W. Neri, C. Monteux, P. Poulin, A. Colin, Giant Electrostrictive Response and Piezoresistivity of Emulsion Templated Nanocomposites, Langmuir. 33 (2017) 4528-4536. doi:10.1021/acs.langmuir.6b04185.

[16] A. Luna, J. Yuan, W. Néri, C. Zakri, P. Poulin, A. Colin, Giant Permittivity Polymer Nanocomposites Obtained by Curing a Direct Emulsion, Langmuir. 31 (2015) 1223112239. doi:10.1021/acs.langmuir.5b02318. 
[17] J. Yuan, A. Luna, W. Neri, C. Zakri, T. Schilling, A. Colin, P. Poulin, Graphene liquid crystal retarded percolation for new high-k materials, Nat. Commun. 6 (2015) 8700. doi:10.1038/ncomms9700.

[18] J.Y. Li, C. Huang, Q. Zhang, Enhanced electromechanical properties in all-polymer percolative composites, Appl. Phys. Lett. 84 (2004) 3124-3126. doi:10.1063/1.1702127.

[19] X. Yin, M. Lallart, P.-J. Cottinet, D. Guyomar, J.-F. Capsal, Mechanical energy harvesting via a plasticizer-modified electrostrictive polymer, Appl. Phys. Lett. 108 (2016) 042901. doi:10.1063/1.4939859.

[20] H. Parant, G. Muller, T. Le Mercier, J.M. Tarascon, P. Poulin, A. Colin, Flowing suspensions of carbon black with high electronic conductivity for flow applications: Comparison between carbons black and exhibition of specific aggregation of carbon particles, Carbon. 119 (2017) 10-20. doi:10.1016/j.carbon.2017.04.014.

[21] S.B. Jones, S.P. Friedman, Particle shape effects on the effective permittivity of anisotropic or isotropic media consisting of aligned or randomly oriented ellipsoidal particles, Water Resour. Res. 36 (2000) 2821-2833. doi:10.1029/2000WR900198.

[22] S. Boisseau, G. Despesse, B. Ahmed, Electrostatic Conversion for Vibration Energy Harvesting, in: M. Lallart (Ed.), Small-Scale Energy Harvest., InTech, 2012. http://www.intechopen.com/books/small-scale-energy-harvesting/electrostatic-conversionfor-vibration-energy-harvesting (accessed September 27, 2017).

[23] M. Lallart, P.-J. Cottinet, L. Lebrun, B. Guiffard, D. Guyomar, Evaluation of energy harvesting performance of electrostrictive polymer and carbon-filled terpolymer composites, J. Appl. Phys. 108 (2010) 034901. doi:10.1063/1.3456084.

[24] P.-J. Cottinet, D. Guyomar, B. Guiffard, C. Putson, L. Lebrun, Modeling and experimentation on an electrostrictive polymer composite for energy harvesting, IEEE Trans. Ultrason. Ferroelectr. Freq. Control. 57 (2010) 774-784. doi:10.1109/TUFFC.2010.1481.

[25] S. Hanson, M. Seok, Y.-S. Lin, Z. Foo, D. Kim, Y. Lee, N. Liu, D. Sylvester, D. Blaauw, A Low-Voltage Processor for Sensing Applications With Picowatt Standby Mode, IEEE J. Solid-State Circuits. 44 (2009) 1145-1155. doi:10.1109/JSSC.2009.2014205.

[26] H. Jayakumar, K. Lee, W.S. Lee, A. Raha, Y. Kim, V. Raghunathan, Powering the internet of things, in: ACM Press, 2014: pp. 375-380. doi:10.1145/2627369.2631644.

[27] V. Raicu, Y. Feldman, eds., Dielectric relaxation in biological systems: physical principles, methods, and applications, First edition, Oxford University Press, Oxford, United Kingdom, 2015. 